\title{
Path Tracking Control for Uncertainty Vehicles via LMI Optimization
}

\author{
Ming Yang, Chang-Fang Chen, Ming-Lei Shu, Yuan-Yuan Yang \\ Shandong Computer Science Center (National Supercomputer Center in Jinan), Shandong Provincial Key Laboratory of \\ Computer Networks, Jinan, China \\ E-mail: chenchf@sdas.org
}

\begin{abstract}
This paper studies the path tracking control of fourwheel steering autonomous vehicles in presence of uncertainties of tire-road condition, modeling uncertainties, and unexpected disturbances. Due to the fast convergence of the wheel subsystem, the simplified vehicle model can be developed based on the singular perturbation. Both the state feedback and the observer-based output feedback controllers are derived using the linear matrix inequalities. The main results rely on a multi-objective approach which can handle various closed-loop specifications, such as disturbance rejection and robust stabilization on different channels within the LMI (Linear Matrix Inequalities) synthesis framework. Simulation results show that the proposed controller can effectively improves the path tracking performance.
\end{abstract}

Keywords-path tracking, four-wheel steering (4WS) vehicles, parameter uncertainty, disturbance attenuation, quadratic stabilization.

\section{INTRODUCTION}

Intelligent vehicle systems have recently become an attractive area of research throughout the world. The aim of the research effort is mainly enhancing driving safety and reducing the driver's workload [1]. Autonomous ground vehicle (AGV) has the great advantages including the improved security, better road utilization, and reduced mobility costs, and thus it has become an emerging research focus worldwide [2]. One of the principal control issues for AGVs is path tracking [3-4]. To guarantee the vehicle lateral stability, it is essential to consider the lateral dynamics for the path tracking control of ground vehicles, which has always been very important and extensively researched.

Although a considerable amount of research on motion control has been reported to ensure path tracking of fourwheel steering (4WS) vehicles during the past couple of decades, such as asymptotic decoupling control [5-6], robust $H_{\infty}$ control [7-8], sliding model control [9], prediction control [10], fuzzy control [11-12], and nonlinear adaptive control [13-14], there still remain certain open problems in this field that are of great theoretical and practical interest. In particular, due to the real-time implementation and external disturbance torque rejection, there currently exists no unified framework for designing user-friendly and cost-effective control scheme for 4WS autonomous vehicles. Furthermore, since some of the system parameters(such as normal load) are even time varying and precisely unmeasurable, those control schemes that strictly rely on such parameters seldom work efficiently in practice [15-17].
This paper proposes a path tracking controller for $4 \mathrm{WS}$ vehicles in the presence of uncertainties of both tire-road condition, modeling uncertainties, and unexpected disturbances. The proposed technique uses multi-objective synthesis approach in the multi-channel spirit. The $H_{\infty}$ norm is used to guarantee the disturbance attenuation and robust stability [18]. First, the state feedback controller is designed, and the corresponding state feedback gain can be obtained the LMI (Linear Matrix Inequalities) optimization. Next, an observer-based output feedback controller is proposed, and the LMI formulations of the closed-loop system specifications and objectives such as disturbance attenuation and quadratic stability are derived for this scenario.

The subsequent parts of this paper are organized as follows. Section 2 presents the system modeling, including: vehicle dynamics, dynamics of path tracking, friction forces, and uncertain vehicle system. Section 3 address the controller design via LMI optimization, where both nonlinear state feedback and observer-based output feedback controllers are proposed. Section 4 presents the simulations and performance evaluation. Finally, we conclude our findings in Section 5.

\section{SYSTEM MODELING}

\section{A. Vehicle Dynamics}

Wherever Times is specified, Times Roman or Times New Roman may be used. If neither is available on your word processor, please use the font closest in appearance to Times. Avoid using bit-mapped fonts if possible. True-Type 1 or Open Type fonts are preferred. Please embed symbol fonts, as well, for math, etc.

The model considered here consists of 7 degrees of freedom (DOF), including longitudinal, lateral motions and yaw motion of the vehicle in addition to the rotational dynamics of the four wheels. The vehicle body-fixed coordinate system is used to set up the model. These are shown in Fig. 1. The governing equations of motion for the vehicle can be expressed as follows:

Longitudinal motion:

$$
m\left(\dot{v}_{x}-\gamma v_{y}\right)=\sum F_{x}-C_{a e r o} v_{x}^{2}
$$

Lateral motion:

$$
m\left(\dot{v}_{y}+\gamma\right)=\sum F_{y}
$$


Yaw motion:

$$
J_{z} \dot{\gamma}=\sum M_{z}
$$

Wheel rotational equations of motion for wheels are as follows:

$$
I_{w j} \dot{w}_{j}=T_{j}-r_{j}\left[\cos \delta_{j} \sin \delta_{j}\right]
$$

where $v_{x}$ denotes the longitudinal vehicle velocity, $v_{y}$ the lateral vehicle velocity, $\gamma$ the yaw rate and $\beta$ the vehicle side slip angle. Notations $m$ and $J_{z}$ are the mass of vehicle and the inertia about $z$ axis, respectively. $C_{\text {aero }}$ represents the coefficient of aerodynamic drag. $I_{w j}$ and $r_{j}$ represent the moment of inertia and the radius of wheel $j . T_{j}$ and $\delta_{j}$ are the wheel torque and wheel steering angle used for the control scheme. $\sum F_{x}, \sum F_{y}, \sum M_{z}$ are the sums of the external forces and moments acting on the vehicle.

$$
\begin{aligned}
\sum F_{x} & =F_{x 1}+F_{x 2}+F_{x 3}+F_{x 4} \\
\sum F_{y} & =F_{y 1}+F_{y 2}+F_{y 3}+F_{y 4} \\
\sum M_{z} & =l_{f}\left(F_{y 1}+F_{y 2}\right)-l_{r}\left(F_{y 3}+F_{y 4}\right) \\
& +l_{d}\left(F_{x 2}-F_{x 1}\right)+l_{d}\left(F_{x 4}-F_{x 3}\right)
\end{aligned}
$$

In the above equations, $F_{x j}, F_{y j}$ and $M_{z j}(j=1,2,3,4)$, defined in the body fixed $x-y-z$ coordinate system, are the external forces and yaw moments mainly resulting from tire/road friction. $l_{f}, l_{r}$ and $l_{d}$ are the distances from the center of gravity to the front axle, the rear axle, and the wheel side.

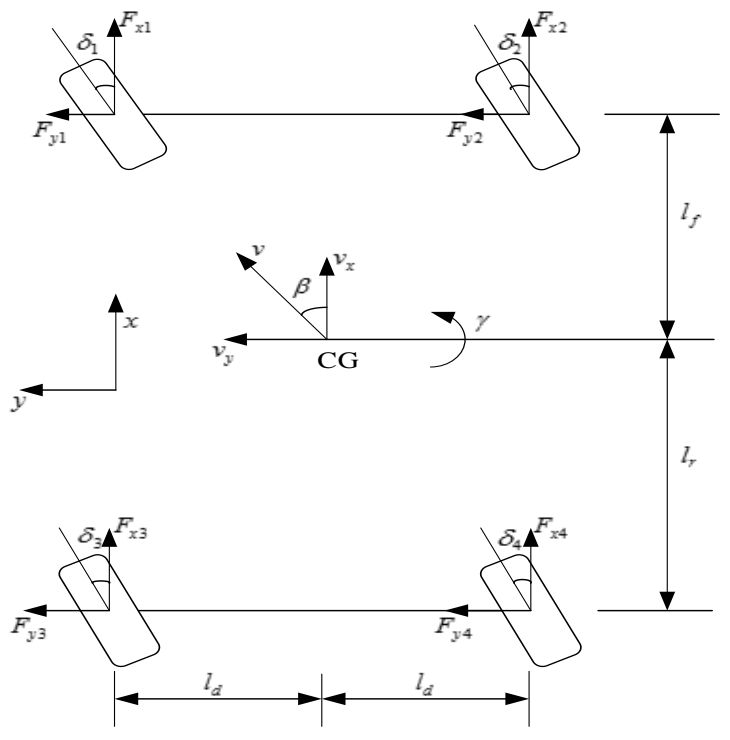

Figure 1. vehicle model

\section{B. Dynamics of Path Tracking}

Because the sensors that measure the lateral deviation are not normally fixed on the vertical line through c.o.g. Furthermore, feedback based on error measured at the c.o.g. leads to bad ride comfort. Hence, it is natural to describe the vehicle dynamics with respect to the lateral displacement at the sensor, i.e., $y_{l}$.

The path tracking dynamics can be expressed as [1]

$$
\begin{aligned}
& \dot{\phi}_{l}=\gamma-\rho_{r e f} v_{x} \\
& \dot{y}_{l}=v_{x}\left(\beta+\phi_{l}\right)+l_{s} \dot{\phi}_{l}
\end{aligned}
$$

where $\phi_{l}$ is the angle between the road centerline and the vehicle longitudinal axis in radians, and $l_{s}$ represents the horizontal distance to the sensor from the $C G . \rho_{\text {ref }}$ is the path curvature.

\section{Friction forces}

The wheel velocities can be derived by two components: the component due to the $C G$ velocity and the component due to the motion about the vertical vehicle axis $z$, i.e.,

$$
\begin{aligned}
& v_{1}=\left[\begin{array}{l}
v_{x 1} \\
v_{y 1}
\end{array}\right]=\left[\begin{array}{l}
v_{x}-l_{d} \gamma \\
v_{y}+l_{f} \gamma
\end{array}\right], \quad v_{2}=\left[\begin{array}{l}
v_{x 2} \\
v_{y 2}
\end{array}\right]=\left[\begin{array}{l}
v_{x}+l_{d} \gamma \\
v_{y}+l_{f} \gamma
\end{array}\right] \\
& v_{3}=\left[\begin{array}{l}
v_{x 3} \\
v_{y 3}
\end{array}\right]=\left[\begin{array}{l}
v_{x}-l_{d} \gamma \\
v_{y}-l_{f} \gamma
\end{array}\right], \quad v_{4}=\left[\begin{array}{l}
v_{x 4} \\
v_{y 4}
\end{array}\right]=\left[\begin{array}{l}
v_{x}+l_{d} \gamma \\
v_{y}-l_{f} \gamma
\end{array}\right]
\end{aligned}
$$

Here, the combined wheel slip is defined according to the Burckhardt approach [2]. The longitudinal slip $S_{L}$ is defined in the direction of the wheel ground contact point velocity $v_{j}, j=1,2,3,4$, and the lateral slip $S_{S}$ at right angles to this.

For braking, the combined wheel slip $\left(v_{r j} \cos \alpha_{j} \leq\left\|v_{w j}\right\|\right)$ :

$$
S_{j}=\left[\begin{array}{c}
\left(v_{r j} \cos \alpha_{j}-\left\|v_{w j}\right\|\right) /\left\|v_{w j}\right\| \\
v_{r j} \sin \alpha_{j} /\left\|v_{w j}\right\|
\end{array}\right]
$$

For driving, the combined wheel slip $\left(v_{r j} \cos \alpha_{j}>\left\|v_{w j}\right\|\right)$

$$
S_{j}=\left[\begin{array}{c}
\left(v_{r j} \cos \alpha_{j}-\left\|v_{w j}\right\|\right) / v_{r j} \cos \alpha_{j} \\
\tan \alpha_{j}
\end{array}\right]
$$

where the tire side slip angle $\alpha_{j}$ is the angle between the wheel plane and the velocity of the wheel ground contact point

$$
\alpha_{j}=\delta_{j}-\beta_{j}, \beta_{j}=\tan \left(v_{y j} / v_{x j}\right)
$$


The resultant slip $S_{\text {Res }}=\left\|S_{j}\right\|$ is directed in the same direction as the resultant friction co-efficient $\mu_{R e s} \cdot \mu_{R e s}$ is a scalar saturation function depending on the magnitude of resultant slip $S_{R e s}$ and road condition. It is known that when $S_{R e s}$ exceeds a threshold associated with road condition, the corresponding $\mu_{\text {Res }}$ saturates and so does the related friction force. Thus, the friction forces in the longitudinal and lateral directions can be expressed as follows

$$
\begin{aligned}
& F_{x j}=F_{z j}\left(\mu_{\text {Res }} \frac{S_{L}}{S_{R e s}} \cos \beta_{j}+\mu_{R e s} k_{S} \frac{S_{S}}{S_{R e s}} \sin \beta_{j}\right) \\
& F_{y j}=F_{z j}\left(\mu_{R e s} k_{S} \frac{S_{S}}{S_{R e s}} \cos \beta_{j}-\mu_{R e s} \frac{S_{L}}{S_{R e s}} \sin \beta_{j}\right)
\end{aligned}
$$

Define

$$
k_{j} \triangleq \frac{\partial \mu_{R e s}}{\partial S_{R e s}}
$$

Then

$$
\left[\begin{array}{l}
F_{x j} \\
F_{y j}
\end{array}\right]=F_{z j}\left[\begin{array}{cc}
\cos \beta_{j} & \sin \beta_{j} \\
-\sin \beta_{j} & \cos \beta_{j}
\end{array}\right]\left[\begin{array}{cc}
k_{j} & 0 \\
0 & k_{S} k_{j}
\end{array}\right]\left[\begin{array}{l}
S_{L j} \\
S_{S j}
\end{array}\right]
$$

where the slope $k_{j}$ in equation (12) depends mainly on road conditions. A better road condition leads to a larger slope $k_{j}$ and in turn provides a larger friction force. Assuming that the vehicle runs on a uniform road condition, and thus $k_{j}$ in equation (12) have the same value as $k$.

\section{Uncertain Vehicle System}

Because the wheel subsystem converges much faster, based on the concept of singular perturbation theory, we can replace the wheel subsystem with its quasi-steady state for model reduction, which yields

$$
S_{L j}=\frac{T_{j}}{F_{z} r_{j} k}, F_{x j}=\frac{T_{j}}{r_{j}}
$$

and using velocity approximation

$$
S_{S j}=\delta_{j}-\beta_{j}=\delta_{j}-\beta-\frac{l_{j}}{v_{0}} \gamma
$$

where $l_{1}=l_{2}=l_{f}, l_{3}=l_{4}=l_{r}$. Take $\left[\begin{array}{ll}T_{j} & \delta_{j}\end{array}\right]^{T}$ as virtual control, and the control structure of wheel torque and steering angle is proposed as

$$
\begin{aligned}
& {\left[\begin{array}{l}
T_{1} \\
\delta_{1}
\end{array}\right]=\left[\begin{array}{c}
0 \\
\beta+l_{f} \gamma / v_{0}
\end{array}\right]+\left[\begin{array}{cc}
F_{z 1} r_{1} k & 0 \\
0 & 1
\end{array}\right]\left[\begin{array}{l}
S_{L 1} \\
S_{S 1}
\end{array}\right]} \\
& {\left[\begin{array}{l}
T_{2} \\
\delta_{2}
\end{array}\right]=\left[\begin{array}{c}
0 \\
\beta+l_{f} \gamma / v_{0}
\end{array}\right]+\left[\begin{array}{cc}
F_{z 2} r_{2} k & 0 \\
0 & 1
\end{array}\right]\left[\begin{array}{l}
S_{L 2} \\
S_{S 2}
\end{array}\right]} \\
& {\left[\begin{array}{l}
T_{3} \\
\delta_{3}
\end{array}\right]=\left[\begin{array}{c}
0 \\
\beta-l_{r} \gamma / v_{0}
\end{array}\right]+\left[\begin{array}{cc}
F_{z 3} r_{3} k & 0 \\
0 & 1
\end{array}\right]\left[\begin{array}{l}
S_{L 1} \\
S_{S 1}
\end{array}\right]} \\
& {\left[\begin{array}{l}
T_{4} \\
\delta_{4}
\end{array}\right]=\left[\begin{array}{c}
0 \\
\beta-l_{r} \gamma / v_{0}
\end{array}\right]+\left[\begin{array}{cc}
F_{z 4} r_{4} k & 0 \\
0 & 1
\end{array}\right]\left[\begin{array}{l}
S_{L 2} \\
S_{S 2}
\end{array}\right]}
\end{aligned}
$$

then the vehicle model is as following:

$$
\begin{aligned}
& \dot{x}=A x+B_{1} w+B_{2} u \\
& z_{\infty}=C_{\infty} x, z_{1}=C_{1} x, z_{u}=u \\
& y=C_{2} x
\end{aligned}
$$

where

$$
\begin{aligned}
& x=\left[\begin{array}{lllll}
\partial v_{x} & \partial v_{y} & \gamma & \phi_{l} & y_{l}
\end{array}\right]^{T}, \\
& u=\left[\begin{array}{llll}
S_{L 1} & S_{S 1} & S_{L 2} & S_{S 2}
\end{array}\right]^{T}, \\
& w=\left[\begin{array}{ll}
f_{w} & \rho_{\text {ref }}
\end{array}\right]^{T}, f_{w}=C_{\text {aero }} v_{0}^{2} / m, \\
& A=\left[\begin{array}{ccccc}
-2 C_{\text {aero }} v_{0} / m & 0 & 0 & 0 & 0 \\
0 & 0 & -1 & 0 & 0 \\
0 & 0 & 0 & 0 & 0 \\
0 & 0 & 1 & 0 & 0 \\
0 & v_{0} & l_{s} & v_{0} & 0
\end{array}\right] \text {, } \\
& B_{1}=\left[\begin{array}{ccccc}
-1 & 0 & 0 & 0 & 0 \\
0 & 0 & 0 & -v_{0} & -l_{s} v_{0}
\end{array}\right]^{T} \text {, } \\
& B_{2}=\left[\begin{array}{ccccc}
b_{11} & 0 & b_{31} & 0 & 0 \\
0 & b_{22} & 0 & 0 & 0 \\
b_{13} & 0 & b_{33} & 0 & 0 \\
0 & b_{24} & 0 & 0 & 0
\end{array}\right]^{T} \text {, } \\
& C_{1}=\left[\begin{array}{lllll}
0 & 0 & 0 & 0 & 1
\end{array}\right], C_{\infty}=I, \\
& C_{2}=\left[\begin{array}{lllll}
0 & 0 & 1 & 0 & 0 \\
0 & 0 & 0 & 0 & 1
\end{array}\right] \text {, } \\
& b_{11}=\frac{\left(F_{z 1}+F_{z 3}\right) k}{m}, b_{13}=\frac{\left(F_{z 2}+F_{z 4}\right) k}{m} \text {, } \\
& b_{22}=\frac{\left(F_{z 1}+F_{z 3}\right) k k_{S}}{m v_{0}}, b_{24}=\frac{\left(F_{z 2}+F_{z 4}\right) k k_{S}}{m v_{0}}, \\
& b_{31}=\frac{-l_{d}\left(F_{z 1}+F_{z 3}\right) k}{J_{z}}, b_{33}=\frac{l_{d}\left(F_{z 2}+F_{z 4}\right) k}{J_{z}}
\end{aligned}
$$

The normal loads $F_{z j}$ on the four wheels, the road condition $k$, and the coefficient of aerodynamic drag $C_{\text {aero }}$ can be expressed as their nominal values plus perturbations: 


$$
\begin{aligned}
& F_{z j}=\bar{F}_{z j}+\Delta F_{z j} \delta_{j}, j=1, \cdots, 4 \\
& k=k_{0}+\Delta k \delta_{5}, \\
& C_{\text {aero }}=\bar{C}_{\text {aero }}+\Delta C_{\text {aero }} \delta_{6}
\end{aligned}
$$

where $\Delta F_{z j}, \Delta k$, and $\Delta C_{\text {aero }}$ are the variation envelopes of $F_{z j}, k$, and $C_{\text {aero }}$, and $\delta_{j}$ are the normalized uncertainties.

The perturbations in matrices $A$ and $B_{2}$ caused by the variations of $\Delta F_{z j}, \Delta k$, and $\Delta C_{\text {aero }}$ can be written as

$$
\begin{aligned}
& A=\bar{A}+\Delta A=\bar{A}+\Xi_{A} \Delta_{A} \Pi_{A} \\
& B_{2}=\bar{B}_{2}+\Delta B_{2}=\bar{B}_{2}+\Xi_{B 2} \Delta_{B 2} \Pi_{B 2}
\end{aligned}
$$

where $\bar{A}$ and $\bar{B}_{2}$ represent the nominal matrices and $\Delta_{A}$ and $\Delta_{B 2}$ are the diagonal matrices with the normalized uncertainties $\delta_{i}$ in the diagonal entries. The detailed results are shown as follows

$$
\begin{aligned}
& \bar{b}_{22}=\frac{\left(\bar{F}_{z 1}+\bar{F}_{z 3}\right) k k_{S}}{m v_{0}}, \bar{b}_{24}=\frac{\left(\bar{F}_{z 2}+\bar{F}_{z 4}\right) k k_{S}}{m v_{0}}, \\
& \bar{b}_{31}=\frac{-l_{d}\left(\bar{F}_{z 1}+\bar{F}_{z 3}\right) k}{J_{z}}, \bar{b}_{33}=\frac{l_{d}\left(\bar{F}_{z 2}+\bar{F}_{z 4}\right) k}{J_{z}}
\end{aligned}
$$

Substituting (19) and (20) into (17), the uncertain vehicle system can be derived as

$$
\begin{aligned}
& \dot{x}=(A+\Delta A) x+B_{1} w+\left(B_{2}+\Delta B_{2}\right) u \\
& z_{\infty}=C_{\infty} x, \quad z_{1}=C_{1} x, \quad z_{u}=u, \quad y=C_{2} x
\end{aligned}
$$

\section{CONTROller Design}

\section{A. State Feedback Control}

Introducing the variables $v=\Sigma z_{\Delta}$, and $z_{\Delta}=$ $\left[\left(\Pi_{A} x\right)^{T}\left(\Pi_{B 2} u\right)^{T}\right]^{T}$, then the uncertain term can be described as

$$
\Delta A x+\Delta B_{2} u=\left[\begin{array}{ll}
\Xi_{A} & \Xi_{B}
\end{array}\right]\left[\begin{array}{ll}
\Delta_{A} & \Delta_{B 2}
\end{array}\right]=E \Sigma z_{\Delta}
$$

where

$$
E=\left[\begin{array}{ll}
\Xi_{A} & \Xi_{B}
\end{array}\right], \quad \Sigma=\operatorname{diag}\left(\Delta_{A}, \Delta_{B 2}\right)
$$

Using the state feedback $u=K x$, and in view of (22), it follows that

$$
\begin{aligned}
& \dot{x}=\left(A+B_{2} K\right) x+B_{1} w+E v \\
& z_{\infty}=C_{\infty} x, z_{1}=C_{1} x, z_{u}=u, y=C_{2} x
\end{aligned}
$$

\section{1) Disturbance Attenuation}

The LMIs for disturbance attenuation of the closed-loop system are first formulated without considering uncertainty. Consider the system

$$
\begin{aligned}
& \dot{x}(t)=A x(t)+B \omega(t) \\
& z(t)=C x(t)+D \omega(t)
\end{aligned}
$$

where $x(t) \in \mathcal{R}^{n}, \omega(t) \in \mathcal{R}^{l}$, and $z(t) \in \mathcal{R}^{p}$ are the states, input and output of the system. Given the constant $\gamma>0$, the system is said to be asymptotically stable and $\left\|T_{z \omega}(s)\right\|_{\infty}<\gamma$ if and only if there exists positive definite matrix $P>0$, and satisfying the following

$$
\left[\begin{array}{ccc}
A^{T} P+P A & P B & C^{T} \\
B^{T} P & -\gamma I_{q} & D^{T} \\
C & D & -\gamma I_{m}
\end{array}\right]<0
$$

Substituting the matrices of the closed-loop system into (25), we obtain the following inequalities

$$
\left[\begin{array}{ccc}
\Sigma_{1} & B_{1} & X C_{\infty}^{T} \\
B_{1}^{T} & -\gamma_{\infty}^{2} I & 0 \\
C_{\infty} X & 0 & -I
\end{array}\right]<0
$$

where $\Sigma_{1}=A X+X A^{T}+B_{2} Y+Y^{T} B_{2}^{T}$, and $X$ is positive definite matrix.

2) Quadratic stabilization

In this section, we consider the quadratic stabilization of the closed-loop system with $w=0$. Quadratic stability of system (24) has the frequency-domain interpretation that the $H_{\infty}$ norm of the transfer function from $v$ to $z_{\Delta}$ is less than one. That is, system (24) is said to be quadratic stabilization if and only if $A+B_{2} K$ is a stable matrix and the transfer function $T_{z_{\Delta} v}$ form $v$ to $z_{\Delta}$ satisfies

$$
\left\|C_{j}\left(S I-A-B_{2} K\right) B_{1}\right\|<0
$$

where $C_{j}=\left[\begin{array}{ll}\Pi_{A}^{T} & \left(\Pi_{B} K\right)^{T}\end{array}\right]$. The LMI formulation of quadratic stabilization can be described as

$$
\left[\begin{array}{cccc}
\Xi_{1} & E & X \Pi_{A}^{T} & Y^{T} \Pi_{B}^{T} \\
E^{T} & -I & 0 & 0 \\
\Pi_{A} X & 0 & -I & 0 \\
\Pi_{B} Y & 0 & 0 & -I
\end{array}\right]<0
$$

with $\Xi_{1}=A X+X A^{T}+B_{2} Y+Y^{T} B_{2}^{T}$.

3) Disturbance attenuation with uncertainty

To guarantee the closed-loop system to be quadratic stable with disturbance attenuation $\gamma_{\infty}$, we consider the plant 


$$
\hat{z}=T(s) \hat{w}
$$

where $\hat{z}^{T}=\left[\begin{array}{ll}z_{\infty}^{T} & z_{\Delta}^{T}\end{array}\right], w^{T}=\left[\begin{array}{ll}w^{T} & v^{T}\end{array}\right]$,

$$
T(s)=\left[\begin{array}{ccc}
A+B_{2} K & \gamma_{\infty}^{-1} B_{1} & E \\
C_{\infty} & 0 & 0 \\
\Pi_{A} & 0 & 0 \\
\Pi_{B} K & 0 & 0
\end{array}\right]
$$

The transfer function $T_{\hat{z} \hat{w}}$ from $\hat{w}$ to $\hat{z}$ is

$$
T_{\hat{z} \hat{w}}=\left[\begin{array}{cc}
\gamma_{\infty}^{-1} T_{z_{\infty} w} & * \\
* & T_{z_{\Delta} v}
\end{array}\right]
$$

It is obvious that $\left\|T_{z_{\infty} w}\right\|<\gamma_{\infty}$ and $\left\|T_{z_{\Delta} v}\right\|<1$ can be both guaranteed if $\left\|T_{\hat{z} \hat{w}}\right\|<1$. We have the following results.

$$
\left[\begin{array}{cccccc}
\Xi_{1} & B_{1} & E & X C_{\infty}^{T} & X \Pi_{A}^{T} & Y^{T} \Pi_{B}^{T} \\
B_{1}^{T} & -\gamma_{\infty}^{2} I & 0 & 0 & 0 & 0 \\
E^{T} & 0 & -I & 0 & 0 & 0 \\
C_{\infty} X & 0 & 0 & -I & 0 & 0 \\
\Pi_{A} X & 0 & 0 & 0 & -I & 0 \\
\Pi_{B} Y & 0 & 0 & 0 & 0 & -I
\end{array}\right]<0
$$

then the state feedback controller is derived by

$$
K=Y^{-1} X
$$

\section{B. Observer-Based Output Feedback Control}

The observer-based output feedback controller can be expressed as

$$
\dot{\hat{x}}=A \hat{x}+B_{2} u+E v+L\left(y-C_{2} \hat{x}\right), \quad u=K \hat{x}
$$

Let $z=\left[\begin{array}{ll}x & e\end{array}\right]$, with $e=x-\hat{x}$, then the closed-loop system is

$$
\dot{z}=\left[\begin{array}{cc}
A+B_{2} K & -B_{2} K \\
0 & A-L C_{2}
\end{array}\right] z+\left[\begin{array}{ll}
B_{1} & E \\
B_{1} & 0
\end{array}\right]\left[\begin{array}{l}
w \\
v
\end{array}\right]
$$

\section{1) Disturbance attenuation}

If there exist a constant matrices $S>0, W$ and a scale $k>0$ such that the following inequality is feasible

$$
\left[\begin{array}{cccc}
\Sigma_{11} & -k P B_{2} K & k P B_{1} & C_{\infty}^{T} \\
-k K^{T} B_{2}^{T} P & \Sigma_{22} & S B_{1} & 0 \\
k B_{1}^{T} P & B_{1}^{T} S & -\gamma_{\infty}^{2} I & 0 \\
C_{\infty} & 0 & 0 & -I
\end{array}\right]<0
$$

where

$$
\begin{aligned}
& \Sigma_{11}=k P A+k A^{T} P+k P B_{2} K+k K^{T} B_{2}^{T} P \\
& \Sigma_{22}=S A+A^{T} S-W C_{2}-C_{2}^{T} W^{T}
\end{aligned}
$$

2) Quadratic stabilization

$$
z_{\Delta}=\left[\begin{array}{l}
\Pi_{A} x \\
\Pi_{B} u
\end{array}\right]=C_{\Delta 1} x+C_{\Delta 2} e
$$

where

$$
C_{\Delta 1}=\left[\begin{array}{c}
\Pi_{A} \\
\Pi_{B} K
\end{array}\right], C_{\Delta 2}=\left[\begin{array}{c}
0 \\
\Pi_{B} K
\end{array}\right]
$$

$$
\left[\begin{array}{cccc}
\Sigma_{11} & -k P B_{2} K & k P E & C_{\Delta 1}^{T} \\
-k K^{T} B_{2}^{T} P & \Sigma_{22} & 0 & C_{\Delta 2}^{T} \\
k E^{T} P & 0 & -I & 0 \\
C_{\Delta 1} & C_{\Delta 2} & 0 & -I
\end{array}\right]<0
$$

The observer gain can be chosen as

$$
L=S^{-1} W
$$

\section{Simulations}

To show the effectiveness of the scheme, simulations are carried out as follows. It is assumed the reference path covered with dry concrete, the curvature of circular path is $\rho_{\text {ref }}=1 / 100 \mathrm{~m}^{-1}$. The desired speed for traveling is $v_{x}=15 \mathrm{~m} / \mathrm{sec}$. The following tire model is employed

$$
\mu_{\text {Res }}\left(\left\|S_{j}\right\|\right)=1.1973\left[1-\exp \left(25.168\left\|S_{j}\right\|\right)\right]-0.5373\left\|S_{j}\right\|
$$

for simulating the dry concrete condition, and the related initial slope (12) is $k=30$. The data of the vehicle system is given as follows; $C_{\text {aero }}=0.3743 \mathrm{~kg} / \mathrm{m}, \quad m=1480 \mathrm{~kg}, \quad J_{z}=$ $1950 \mathrm{kgm}^{2}, l_{f}=1.421 \mathrm{~m}, l_{l}=1.029 \mathrm{~m}, l_{d}=0.751 \mathrm{~m}, k_{s}=0.9, \quad g=$ $9.81 \mathrm{~m} / \mathrm{s}^{2}$. The control inputs are the front-wheel steering angles and the desired wheel torques.

The vehicle control system is assumed to start with the following initial state: $v_{x}=15 \mathrm{~m} / \mathrm{sec}, \quad v_{y}(0)=0 \mathrm{~m} / \mathrm{sec}, \quad \gamma(0)=$ $0 \mathrm{deg} / \mathrm{sec}, y_{c}(0)=0.2 m, \phi_{l}(0)=0 \mathrm{deg}$. Fig. 2 depicts the result of the plane motion of the vehicle under the state feedback control algorithm. It can be seen that the vehicle approaches the reference path in a slightly swinging manner, and its path 
eventually overlaps the reference path although there exist parameter uncertainties. Fig. 3 illustrates the vehicle states with the observer-based output feedback controller. It is shown that the states of the controlled system perform asymptotically convergent to the ideal states, i.e., the vehicle can track the desired curved path.
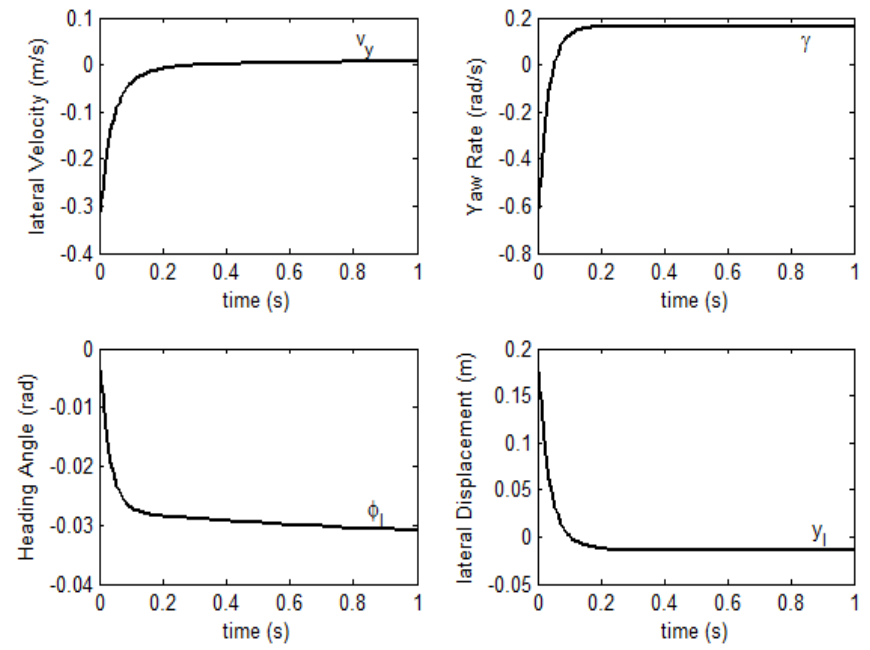

Figure 2. Response of vehicle states (state feedback)
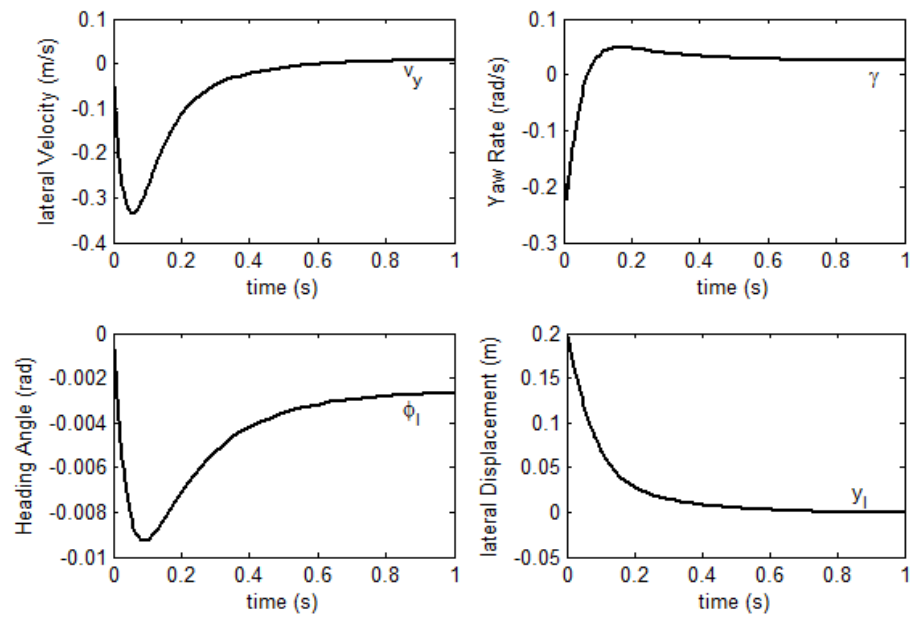

Figure 3. Response of vehicle states (observer-based output feedback)

\section{CONCLUSION}

A path tracking control scheme is presented for 4WS vehicles in the presence of uncertainties of modeling uncertainties, tire-road conditions, and unexpected disturbances. Both the state feedback controller and observer-based output feedback controller are designed via LMI optimization technique. The main results rely on the multi-objective synthesis approach. The proposed solution are subject to some conservatism induced by one single Lyapunov matrix enforcing all the specifications and the special structure of the controller. However, the designed controller can provide satisfactory disturbance attenuation performance and robustly stabilize the uncertain vehicle system. Simulation results are included to validate the proposed control scheme.

\section{ACKNOWLEDGMENT}

This work was supported by the NSFC (National Natural Science Foundation of China), Grant No. 61603224.

\section{REFERENCE}

[1] N. MinoiuEnache, M. Netto, S. Mammar, B. Lusetti, James Lam. Driver steering assistance for lane departure avoidance, Engineering Practice 2009, 17:642-651.

[2] U. Kiencke, L. Nielsen. Automotive Control Systems For Engine, Driveline, and Vehicle. Berlin: Springer-Verlag 2005.

[3] N. M. Enache, S. Mammar, M. Netto, and B. Lusetti. Driver steering assistance for lane-departure avoidance based on hybrid automata and composite Lyapunov function, IEEE Trans. on Intelligent Transportation Systems 2010, 11(1):28-39.

[4] V. Cerone, M. Milanese, D. Regruto. Combined automatic lanekeeping and driver's steering through a 2-DOF control strategy, IEEE Trans. on control systems technology. 2009, 17(1):135-142.

[5] R. Marino and S. Scalzi, Asymptotic sideslip angle and yaw rate decoupling control in four-wheel steering vehicles, Vehicle System Dynamics, 2010, 48(9): 999-1019.

[6] C. Chen, Y. Jia, Q. Gao, F. Yu, Asymptotic decoupling control with active front steering and electronic differentials in four wheel steering vehicles, CCC 2011, Yantai, China, 2011,pp. 111-116.

[7] Y. Jia, Robust control with decoupling performance for steering and traction of 4WS vehicles under velocity-varying motion, IEEE Trans. on Control Systems Technology, 2000, 8(3):554-569.

[8] C. Chen, Y. Jia, J. Du, F. Yu, Lane keeping control for autonomous 4WS4WD vehicles subject to wheel slip constraint, ACC 2012, Montreal, Canada, 6515-6520, 2012.

[9] M. Amodeo, A. Ferrara, R. Terzaghi, and C. Vecchio, Wheel slip control via second-order slidingmode generation, IEEE Trans. on Intelligent Transportation Systems, 2010, 11(1), pp. 122-131.

[10] G. V. Raffo, G. K. Gomes, J. E. Normey-Rico, C. R. Kelber. A predictive controller for autonomous vehicle path tracking, IEEE Trans. on Intelligent Transportation Systems, 2009, 10(1): 92-102.

[11] J. Naranjo, C. Gonzalez, R. Garcia. Lane-change fuzzy control in autonomous vehicles for the overtaking maneuver, IEEE Trans. on Intelligent Transportation Systems, 2008, 9(3): 438-450.

[12] J. Naranjo, C. Gonzalez, J. Reviejo, R. Garcia, and T. de Pedro, Adaptive fuzzy control for inter-vehicle gap keeping, IEEE Trans. on Intelligent Transportation Systems, 2003, 4(3): 132-142.

[13] C. Chen, Y. Jia, M. Shu, Y. Wang, Hierarchical adaptive pathtracking control for autonomous vehicles, IEEE Transactions on Intelligent Transport Systems, 2015, 16(5): 2900-2912.

[14] C. Chen, M. Shu, R. Liu, Virtual-point-based asymptotic tracking control of 4WS vehicles, International Journal of Control, Automation, and Systems, 2015, 13(2): 371-378.

[15] J. Ackermann, J. Guldner, and W. Sienel, Linear and nonlinear controller design for robust automatic steering, IEEE Transactions on Control Systems Technology, 1995, 3(1):132-143.

[16] S. Kumarawadu, and T. T. Lee, Neuroadaptive output tracking of fully autonomous road vehicles with an observer, IEEE Transactions on Intelligent Transportation Systems, 2006, 7(4):335-345.

[17] S. Kumarawadu, T. T. Lee, Neuroadaptive combined lateral and longitudinal control of highway vehicles using RBF networks, IEEE Transactions on Control Systems Technology, 2006, 7(4):500-512.

[18] C. Scherer, P. Gahinet, M. Chilali, Multiobjective Output-Feedback Control via LMI Optimization, IEEE Transaction on Automatic Control, 1997, 42(7):896-911. 


$$
\begin{aligned}
& \bar{A}=\left[\begin{array}{ccccc}
-2 \bar{C}_{\text {aero }} v_{\mathrm{o}} / m & 0 & 0 & 0 & 0 \\
0 & 0 & -1 & 0 & 0 \\
0 & 0 & 0 & 0 & 0 \\
0 & 0 & 1 & 0 & 0 \\
0 & v_{0} & l_{s} & v_{0} & 0
\end{array}\right], \\
& \bar{B}_{2}=\left[\begin{array}{cccc}
\bar{b}_{11} & 0 & \bar{b}_{13} & 0 \\
0 & \bar{b}_{22} & 0 & \bar{b}_{24} \\
\bar{b}_{31} & 0 & \bar{b}_{33} & 0 \\
0 & 0 & 0 & 0 \\
0 & 0 & 0 & 0
\end{array}\right], \quad \Xi_{A}=\left[\begin{array}{ccccc}
-2 v_{0} / m & 0 & 0 & 0 & 0 \\
0 & 0 & -1 & 0 & 0 \\
0 & 0 & 0 & 0 & 0 \\
0 & 0 & 1 & 0 & 0 \\
0 & v_{0} & l_{s} & v_{0} & 0
\end{array}\right] \text {, } \\
& \Delta_{A}=\operatorname{diag}\left(\delta_{6}, 1,1,1,1\right), \quad \Pi_{A}=\operatorname{diag}\left(\Delta C_{\text {aero }}, 1,1,1,1\right) \text {, } \\
& \Xi_{B 2}=\left[\begin{array}{ccccc}
\frac{2}{m} & \frac{2 k_{S}}{m v_{0}} & 0 & 0 & 0 \\
\frac{2}{m} & \frac{2 k_{S}}{m v_{0}} & 0 & 0 & 0 \\
\vdots & \vdots & \vdots & \vdots & \vdots \\
\frac{2}{m} & \frac{2 k_{S}}{m v_{0}} & 0 & 0 & 0
\end{array}\right]^{T}, \\
& \Delta_{B 2}=\operatorname{diag}\left(\delta_{1}, \delta_{2}, \delta_{3}, \delta_{4}, \delta_{5}, \delta_{1} \delta_{5}, \delta_{2} \delta_{5}, \delta_{3} \delta_{5}, \delta_{4} \delta_{5}\right) \text {, } \\
& \Pi_{B 2}=\left[\begin{array}{ll}
\Pi_{B 2}^{1 T} & \Pi_{B 2}^{2 T}
\end{array}\right]^{T} \text {, } \\
& \Pi_{B 2}^{1}=\left[\begin{array}{cc}
\Delta F_{z 1} \bar{k} & \Delta F_{z 1} \bar{k} \\
O & O \\
\Delta F_{z 3} \bar{k} & \Delta F_{z 3} \bar{k} \\
O & 0 \\
\left(\bar{F}_{z 1}+\bar{F}_{z 3}\right) \Delta k & \left(\bar{F}_{z 1}+\bar{F}_{z 3}\right) \Delta k \\
\Delta F_{z 1} \Delta k & \Delta F_{z 1} \Delta k \\
O & O \\
\Delta F_{z 3} \Delta k & \Delta F_{z 3} \Delta k \\
0 & 0
\end{array}\right]^{T}, \\
& \Pi_{B 2}^{2}=\left[\begin{array}{cc}
\mathrm{O} & \mathrm{O} \\
\Delta F_{z 2} \bar{k} & \Delta F_{z 2} \bar{k} \\
\mathrm{O} & \mathrm{O} \\
\Delta F_{z 4} \bar{k} & \Delta F_{z 4} \bar{k} \\
\left(\bar{F}_{z 2}+\bar{F}_{z 4}\right) \Delta k & \left(\bar{F}_{z 2}+\bar{F}_{z 4}\right) \Delta k \\
\mathrm{O} & \mathrm{O} \\
\Delta F_{z 2} \Delta k & \Delta F_{z 2} \Delta k \\
\mathrm{O} & \mathrm{O} \\
\Delta F_{z 4} \Delta k & \Delta F_{z 4} \Delta k
\end{array}\right]^{T}, \\
& \bar{b}_{11}=\frac{\left(\bar{F}_{z 1}+\bar{F}_{z 3}\right) k}{m}, \bar{b}_{13}=\frac{\left(\bar{F}_{z 2}+\bar{F}_{z 4}\right) k}{m} \text {, }
\end{aligned}
$$

